\title{
Diagnostics of local and global coronal magnetic field using global coronal waves
}

\author{
Istvan Ballai \\ SPARG, Department of Applied Mathematics, University of Sheffield, Hounsfield Road, Hicks \\ Building, Sheffield, S3 7RH, UK \\ email: i.ballai@sheffield.ac.uk
}

\begin{abstract}
Large scale eruption events in the solar atmosphere can generate global waves, i.e. waves which propagate over distances comparable to the solar radius. In the low solar corona, global waves observed by SOHO/EIT, generated by coronal mass ejections or flares are usually referred to as EIT waves. Since EIT waves carry information about their environment, they can be used for diagnostics of, e.g. the local and global magnetic field. This contribution presents theoretical models for finding values of magnetic field in the quiet Sun and coronal loops, and explores the physical connection between local and global solar coronal events (e.g. flares and coronal loop oscillations).
\end{abstract}

Keywords. Sun: corona, Sun: magnetic fields, waves

\section{EIT waves and coronal global seismology}

Coronal seismology is one of the newest branches of solar physics that emerged after the first direct observational detection of magnetic oscillations in the solar corona (Roberts, Edwin and Benz (1984); Nakariakov et al. (1999)). Many of the basic physical parameters of the Sun's outer atmosphere, the corona, cannot be measured directly (e.g. magnetic field, transport coefficients). The solar atmosphere, however, is dominated by magnetism therefore the determination of coronal magnetic field, the parameter which controls the dynamics, the structure and evolution of the corona of the Sun, has been a challenge for solar physicists for several decades. The vacuum-like nature of the corona means that measurements of field strength, as are available in sunspots through the Zeeman or Hanle effects, are not possible in the corona. Coronal seismology is using the observable properties of waves to determine plasma and field parameters in the corona.

Unambiguous evidence for large-scale coronal impulses initiated during the early stage of a flare and/or CME has been provided by Extreme-ultraviolet Imaging Telescope (EIT) observations onboard SOHO and by TRACE/EUV. EIT waves propagate in the quiet Sun with speeds of $250-400 \mathrm{~km} \mathrm{~s}^{-1}$ at an almost constant altitude. EIT waves have two stages: first there is an early (driven) stage where the wave correlates to radio type II bursts due to the creation of an energized population which serves as the source of radio emission. The second stage consists of a freely propagating wavefront which is observed to interact with coronal loops, see, e.g.Will-Davey \& Thompson (1999) and Harra \& Sterling (2003). Using TRACE/EUV 195 Å observations, Ballai, Erdélyi \& Pintér (2005) have shown that EIT waves (seen in this wavelength) are waves with average periods of the order of $400 \mathrm{~s}$. Since at this height, the magnetic field can be considered vertical, EIT waves were considered fast MHD waves (FMW).

The event which occurred on 13 June 1998 and studied by Will-Davey \& Thompson (1999) and Ballai, Erdélyi \& Pintér (2005) shows an EIT wave propagating northward and interacting with large coronal loops. This interaction resulted in large transversal 
loop oscillations identified as fast kink oscillations which can be used to estimate the magnetic field in the coronal loop (Nakariakov et al. (1999)) or the energy of the EIT wave generating the oscillation. Ballai, Erdélyi \& Pintér (2005) were able estimate that the minimum energy of the EIT wave in the event occurred on 13 June 1998 was $3.8 \times 10^{18}$ $\mathrm{J}$, a value obtained after applying the formula

$$
E=\frac{\pi L\left(\rho_{i} R^{2}+\rho_{e} / \lambda_{e}^{2}\right)}{2}\left(\frac{x_{\max }-x_{1}}{t_{\max }-t_{1}}\right)^{2},
$$

where $L$ is the length of the loop, $R$ the radius, $\rho_{i}$ and $\rho_{e}$ are the densities inside and outside the loop, $x_{\max }$ is the maximum deflection of the tube which occurs at $t_{\max }$ and $x_{1}$ is an intermediate deflection which occurs at the moment $t_{1}$ and $\lambda_{e}^{-1}$ the decay length of perturbations outside the cylinder. This idea can be inverted: if, somehow, we found the energy of global EIT waves, using Eq. (1.1) we would be able to derive the Alfvén speed in the loop (i.e. the magnetic field), density ratio, geometrical sizes of the loop, i.e. global waves can be used for local coronal seismology.

EIT waves propagating in a plasma of density $\rho$ can be used to obtain information about the generating flare. Supposing that the initial blast wave decayed into a FMW when reaching the FOW of the TRACE instrument, the energy of the flare can be estimated from the Sedov-Taylor relation as

$$
E_{f}=\left(d^{5} \rho\right) / \tau^{2},
$$

where $d$ is the distance travelled by the blast wave, $\tau$ the time required to cover the distance $\tau$. For the mentioned event, we obtain that $d=285.7 \mathrm{Mm}$ and $\tau=1264 \mathrm{~s}$, so the energy of the source was $E_{f}=1.7 \times 10^{24} \mathrm{~J}$.

The difference between $E_{f}-E$ is an energy lost due to the geometrical damping of EIT waves but also due to some physical damping mechanisms which may act in the quite Sun (e.g. viscosity, thermal conduction, etc.). Using this energy difference we can estimate an average value of the transport coefficient for the quiet Sun.

EIT waves propagating perpendicular to the ambient magnetic field, their dispersion relation allows us to determine the magnetic field of the quiet Sun. We assume $1.4 \times 10^{6} \mathrm{~K}$ as a representative intermediate temperature value at which EIT waves propagate. The sound speed, $c_{S}$, corresponding to this temperature is $179 \mathrm{~km} \mathrm{~s}^{-1}$. Since the FMWs propagate perpendicular to the field, their phase speed is given by $v_{E I T}=\left(c_{S}^{2}+v_{A}^{2}\right)^{1 / 2}$, where $v_{A}$ is the Alfvén speed. Through the Alfvén speed we can obtain the intensity of the magnetic field as $B=4 \times 10^{-13} \sqrt{n\left(v_{E I T}^{2}-c_{S}^{2}\right)}$, where the number density of the plasma, $n$, is given in $\mathrm{m}^{-3}$, and the propagation speeds in $\mathrm{m} \mathrm{s}^{-1}$. Supposing an average density of $3 \times 10^{14} \mathrm{~m}^{-3}$, for an EIT wave propagating with speeds of $250-400 \mathrm{~km} \mathrm{~s}^{-1}$ we obtain magnetic fields of $1.2-2.47 \mathrm{G}$.

\section{Acknowledgements}

I. Ballai acknowledges the financial support by Nuffield Foundation (NAL/00746/G) and the NFS Hungary (OTKA, T043741)

\section{References}

Ballai, I, Erdélyi, R. \& Pintér, B 2005, ApJ (Letters) 633, L145

Harra, L.K. \& Sterling, A.C. 2003, ApJ, 587, 429

Nakariakov, V.M., Ofman, L., DeLuca, E.E., Roberts, B. \& Davila, J.M. 1999, Science, 285, 862

Roberts, B, Edwin, P.M. \& Benz, A.O. 1984, ApJ, 279, 857

Wills-Davey, M.J. \& Thompson, B.J. 1999, Sol Phys., 190, 467 